Notas Clínicas

\title{
Carcinomatosis peritoneal mucinosa tratada con cisplatino (CDDP) intraperitoneal y 5-FU endovenoso
}

C. Bosch Roig ${ }^{1}$, E. Roselló Sastre ${ }^{2}$, D. Almenar Cubells ${ }^{1}$, E. Grau Cardona ${ }^{3}$, C. Molins Palau ${ }^{1}$ T. Ripollés Cruzales ${ }^{4}$

\section{Resumen}

- Propósito: describir el tratamiento de una entidad clínica poco frecuente de curso clínico variable.

- Material y método: se presenta el caso de un paciente con carcinomatosis peritoneal mucinosa, variante intermedia.

- Resultados y conclusiones: una cirugía de citoreducción adecuada, seguida de quimioterapia con CDDP Intraperitoneal (IP) y 5FU ev, puede lograr una larga supervivencia en pacientes afectos de carcinomatosis peritoneal, con buena tolerancia al tratamiento.

Palabras clave:

Pseudomixoma peritonei. Quimioterapia intraperitoneal. Cirugía.

Oncología, 2005; 28 (4):202-206

\footnotetext{
${ }^{1}$ Unidad de Oncología Medica

${ }^{2}$ Servicio de Anatomía Patológica

${ }^{3}$ Servicio de Cirugía General y Digestiva

${ }^{4}$ Servicio de Radiodiagnóstico
}

Hospital Universitario Dr. Peset

Valencia 


\section{Summary}

- Purpose: To describe the treatment of a slightly frequent clinical entity of variable clinical course.

- Material and methods: We present the case of a patient with mucinous peritoneal carcinomatosis, intermediate variant.

- Results and conclusion: A suitable cytoreductive surgery, followed by chemotherapy with intraperitoneal CDDP and intravenous 5-FU, can achieve a long survival of patients with peritoneal carcinomatosis, with good tolerance for the treatment.

Key words: Pseudomyxoma peritonaei. Intraperitoneal chemotherapy. Cytoreductive surgery.

El Pseudomixoma peritonei es una entidad rara, $(0,2-0,5 \%$ de todas las neoplasias del tracto gastrointestinal $)^{1,2}$ que se manifiesta como una "ascitis mucinosa" en la que se encuentra abundante material mucinoso intraperitoneal libre o implantado sobre las serosas y estando generalmente asociado a tumores apendiculares, aunque también se han descrito casos asociado de otras localizaciones en abdomen y pelvis, como colon u ovario. Está considerado como la diseminación peritoneal de tumores de comportamiento biológico variable, que va desde tumores benignos apendiculares (cistoadenomas mucinosos) a verdaderos adenocarcinomas mucinosos de colon o estómago, mostrando la misma entidad una evolución distinta. Así se ha intentado clasificar el pseudomixoma atendiendo a sus características morfológicas, independientemente de su origen, en adenomucinosis diseminada peritoneal (DPAM), carcinomatosis mucinosa peritoneal (PMCA) y una entidad intermedia, que es la carcinomatosis mucinosa peritoneal de características intermedias o discordantes (PMCA-I/D) de Ronnet y Sugarbaker ${ }^{3}$. En el primer caso, los implantes mucinosos son escasos, estando constituidos principalmente por moco, con escaso componente epitelial muy bien diferenciado, mientras que en el PMCA, el componente epitelial es mayor y con claros rasgos de malignidad. En el tercer grupo, suele haber un predominio del material mucinoso sobre el epitelial, pero se aprecia ya rasgos displásicos.

El tratamiento en las dos ultimas décadas pretende lograr una citoreducción quirúrgica máxima, es decir conseguir una enfermedad residual menor de $2,5 \mathrm{~mm}$. Y, después, administrar un tratamiento intraperitoneal con Mitomicina-C y 5FU, como fárma- cos básicos, con o sin técnica de hipertermia ${ }^{4-7}$. También se han utilizado, Cisplatino (CDDP) y Ciclofosfamida. De esta forma se están consiguiendo supervivencias mas prolongadas.

\section{Caso clínico}

Varón de 66 años con antecedentes de obesidad, hipertensión arterial, hernia umbilical. Portador de una prótesis en rodilla izquierda.

Consulta por dolor abdominal en fosa iliaca izquierda de 1 semana de evolución y de intensidad progresiva.

Exploraciones complementarias:

- Enema opaco: diverticulosis en colon descendente.

- CEA: 2,4; CA19.9: 1,2

- TAC (Fig. 1) y Ecografía Abdominal (Fig. 2):

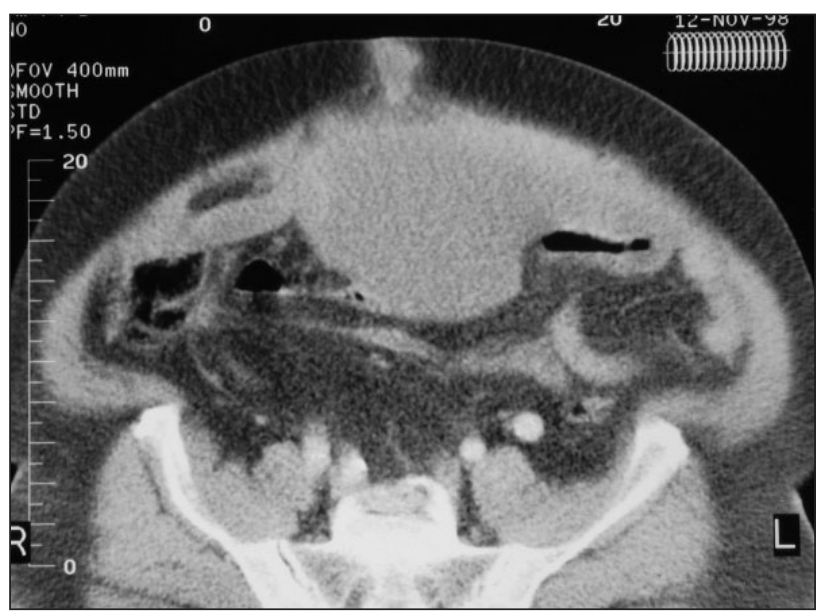

Figura 1. 


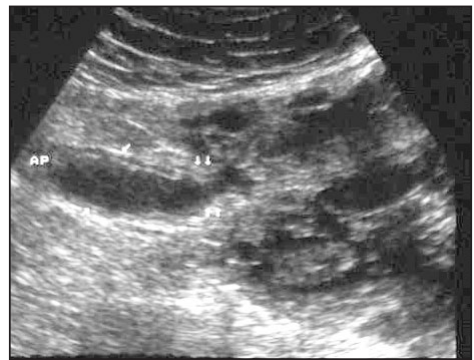

Figura 2.

Ascitis loculada en mesogastrio, vacío derecho y región perihepática. Nódulos peritoneales compatibles con carcinomatosis peritoneal mucocele en apéndice.

Con el diagnostico radiológico de Pseudomixoma peritoneal, se le interviene el 26-11-1998. Intraoperatoriamente se describe ascitis mucoide con varios implantes en epiplón mayor, cara posterior gástrica, otros en peritoneo parietal derechos e izquierdos y reflexión vesicoperitoneal. Tumoración en apéndice vermicular exteriorizada de 10 x 6 × $3 \mathrm{~cm}$. Se practica exéresis de todos implantes peritoneales y una colectomía derecha. Se dejó un catéter de Tenckoff en fondo de saco Douglas.

El estudio del apéndice identifica una masa quística de $8 \times 6 \times 3 \mathrm{~cm}$ repleta de moco junto con implantes mucinosos en peritoneo, de un tamaño que oscilaban entre 0,5 y $7 \mathrm{~cm}$. El diagnóstico histológico fue de Cistoadenocarcinoma mucinoso bien diferenciado de apéndice, con infiltración focal parietal y con Carcinomatosis peritoneal mucinosa, variante intermedia, dado que los lagos de moco mostraron

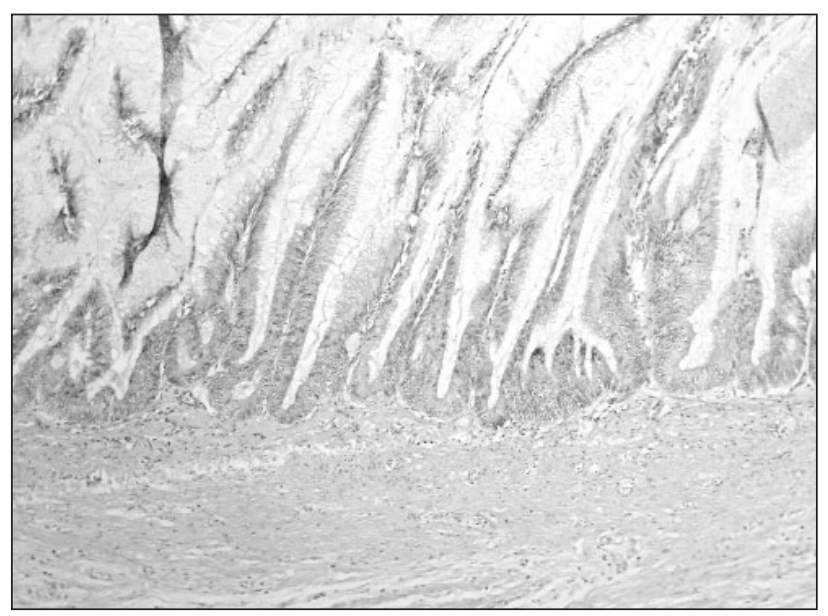

Figura 3.

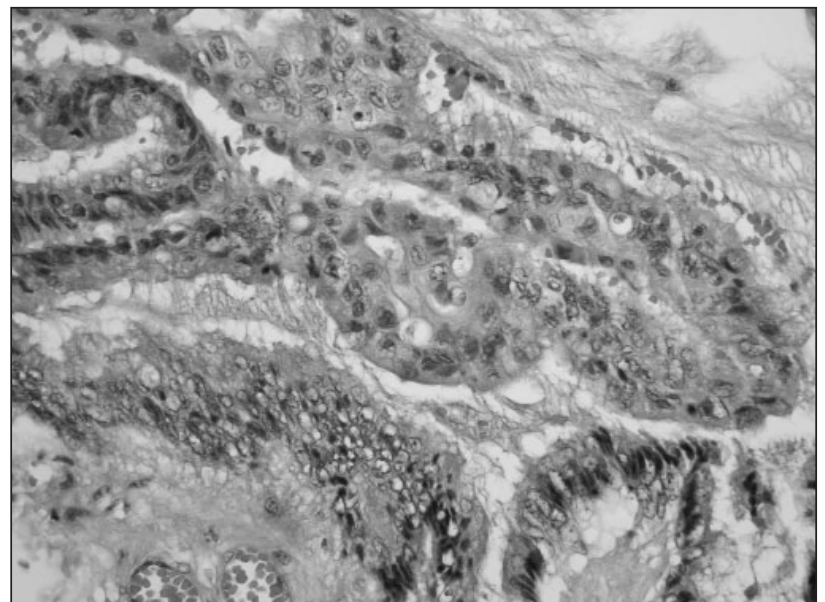

Figura 4.

un rico revestimiento epitelial, con atipias focales. (Fig. 3: Imagen histológica de la tumoración apendicular. Fig. 4: Imagen histológica de uno de los implantes en pared gástrica).

Un mes después de la intervención inició quimioterapia intraperitoneal (Qt IP) de CDDP a dosis de $100 \mathrm{mg} / \mathrm{m}^{2}$ en $100 \mathrm{cc}$ de suero fisiológico en 4 horas, con prehidratación previa habitual; y $5 \mathrm{FU}$ en infusión continua de 5 días vía endovenosa a dosis de $1000 \mathrm{mg} / \mathrm{m}^{2}$ y día, cada 4 semanas. Recibió 6 ciclos del mencionado esquema. Se redujo la dosis al $75 \%$ por insuficiencia renal de grado 1 . Además, presentó anemia $2^{\circ}$ y neutropenia $3^{\circ}$ según los criterios de la OMS, por lo que se aplazó una semana el quinto ciclo de quimioterapia.

El TAC abdominopélvico después del tercer ciclo fue normal.

Posteriormente ha seguido controles en nuestro servicio mediante estudios de TAC abdominal-torácico y CEA y CA19.9 permaneciendo sin signos de progresión, con un seguimiento de 65 meses.

\section{Discusión}

La citoreducción máxima y las características histológicas de los implantes que produce esta entidad clínica, son los dos factores pronósticos mas importantes del Pseudomixoma peritonei. Esto mismo se comunica en el estudio de Ronnett $\mathrm{BM}$ et $\mathrm{al}^{4}$, en el que la supervivencia a los 5 años y 10 años de los pacientes con adenomas mucinosos de apéndice y con implantes mucosos con escasas células y po- 
cas atipias (denominada adenomucosis diseminada peritoneal o DPAM) es del $75 \%$ y $68 \%$ respectivamente. En cambio, en los pacientes con carcinomatosis mucosa peritoneal con implantes epiteliales con atipias francas y células en anillo de sello, la supervivencia baja al $14 \%$ y $3 \%$ a los 5 y 10 años respectivamente.

Existe una entidad mixta en esta clasificación llamada carcinomatosis mucinosa peritoneal de características intermedias, que seria el caso que comunicamos que, en la serie de Sugarbaker et $\mathrm{al}^{5}$ tiene un pronóstico semejante al PMCA (carcinomatosis mucosa peritoneal), aunque continúa habiendo controversia en la clasificación de esta entidad, así como en la terminología empleada.

En cuanto a la citoreducción, Olivier Glehen ${ }^{6}$, ha publicado recientemente una serie de pacientes con adenocarcinoma mucinoso en los no que se lograba una resección máxima de la enfermedad, cayendo entonces la supervivencia media a 16,4 meses. En el caso que describimos se ha logrado una larga supervivencia, de más de 65 meses, que se puede justificar al conseguir una cirugía adecuada, a pesar de pertenecer e la clasificación de Ronnet y Sugarbaker al grupo pronóstico intermedio o PMCA-I/D.

La técnica de Qt IP que utilizamos en nuestro caso, es la más reproducible con mejor tolerancia, aunque con menos garantías teóricas de distribución uniforme del fármaco por el peritoneo; ya que la Qt IP con hipertermia puede llegar a tener una morbilidad grave del $27 \%$ y una mortalidad del $2,7 \%{ }^{5}$.

El CDDP ha mostrado eficacia vía IP en los tumores epiteliales de ovario, en cambio en los tumores mucinosos apendiculares su experiencia es más limitada. Se ha utilizado también en dos series italianas, de 33 y 132 pacientes $^{8,9}$ y una alemana ${ }^{10}$ de 13 pacientes, que se administraba junto a Mitomicina $\mathrm{C}$, pero con técnica de hipertermia; pero esta última presentaba una mortalidad excesiva en los primeros 90 días que, en el caso de la serie alemana ${ }^{10}$, llegaba al $15 \%$. Esto contrasta con la mínima toxicidad presentada en el caso que describimos.

La quimioterapia sistémica se ha utilizado en pacientes con enfermedad metastásica y en los que no se ha logrado una citoreducción adecuada como en la serie de Olivier Glehen ${ }^{6}$ en la que los fármacos administrados por vía sistémica fueron también Mitomicina-C y $5 \mathrm{FU}$, sin embargo, no se comunican los resultados obtenidos.
En dos series japonesas ${ }^{11,12}$ (en forma de resúmen), de dos y cinco casos en las que se utilizaron, al igual que en nuestro paciente, varios ciclos postoperatorios de $5 \mathrm{FU}$ en infusión continua vía endovenosa, junto al CDDP IP, se comunican dos casos con supervivencias de 44 y 46 meses. Por lo que pensamos que, sean cuales sean las características histológicas de las lesiones de una carcinomatosis peritoneal con cirugía de citoreducción adecuada, seguida de quimioterapia con CDDP IP y 5FU ev se puede lograr una larga supervivencia, con muy buena tolerancia al tratamiento.

\author{
Correspondencia: \\ Dr. C. Bosch Roig \\ Unidad de Oncología Médica \\ Hospital Universitario Dr. Peset \\ Av. Gaspar Aguilar, 90 \\ E-46017 Valencia \\ E-mail: bosch_car@gva.es \\ oncologia_peset@gva.es
}




\section{Bibliografía}

1. Lyss AP. Appendiceal malignancies. Semin Oncol. 1988 Apr;15(2):129-37

2. McCusker ME, Cote TR, Clegg LX, Sobin LH. Primary malignant neoplasms of the appendix: a population-based study from the surveillance, epidemiology and end-results program, 1973-1998. Cancer. 2002 Jun 15;94(12):3307-12.

3. Ronnett BM, Zahn CM, Kurman RJ, Kass ME, Sugarbaker PH, Shmookler BM. Disseminated peritoneal adenomucinosis and peritoneal mucinous carcinomatosis. A clinicopathologic analysis of 109 cases with emphasis on distinguishing pathologic features, site of origin, prognosis, and relationship to "pseudomyxoma peritonei". Am J Surg Pathol. 1995 Dec;19(12):1390-408.

4. Ronnett BM, Yan H, Kurman RJ, Shmookler BM, Wu L, Sugarbaker PH. Patients with pseudomyxoma peritonei associated with disseminated peritoneal adenomucinosis have a significantly more favorable prognosis than patients with peritoneal mucinous carcinomatosis. Cancer. $2001 \mathrm{Jul}$ 1;92(1):85-91.

5. Sugarbaker PH, Chang D. Results of treatment of 385 patients with peritoneal surface spread of appendiceal malignancy. Ann Surg Oncol. 1999 Dec;6(8):727-31.

6. Glehen O, Mohamed F, Sugarbaker PH. Incomplete cytoreduction in 174 patients with peritoneal carcinomatosis from appendiceal malignancy. Ann Surg. 2004 Aug;240(2):27885.

7. Shen P, Levine EA, Hall J, Case D, Russell G, Fleming R, McQuellon R, Geisinger KR, Loggie BW. Factors predicting survival after intraperitoneal hyperthermic chemothe- rapy with mitomycin $\mathrm{C}$ after cytoreductive surgery for patients with peritoneal carcinomatosis. Arch Surg. 2003 Jan;138(1):26-33.

8. Scuderi S, Costamagna D, Vaira M, Barone R, De Simone $\mathrm{M}$. [Treatment of pseudomyxoma peritonei using cytoreduction and intraperitoneal hyperthermic chemotherapy] [Article in Italian]. Tumori. 2003 Jul-Aug;89(4 Suppl):43-5.

9. Deraco M, Kusamura S, Gronchi A. [Cytoreductive surgery (peritonectomy) and intraperitoneal hyperthermic chemotherapy: an innovative and effective approach to the treatment of pseudomyxoma peritonei] [Article in Italian]. Tumori. 2003 Jul-Aug;89(4 Suppl):54-5. Italian.

10. Piso P, Werner U, Bektas H, Kubicka S, Manns MP, Klempnauer J. [Peritoneal carcinosis in appendiceal carcinoma-at what price can the prognosis be improved?] [Article in German]. Dtsch Med Wochenschr. 2000 Oct 27;125(43):1283-7.

11. Sasaki A, Terashima M, Okamoto K, Ikeda K, Takagane A, Takiyama I, Shimada Y, Yamamoto M, Nakajima J, Sasaki N, Saito K. [Intraperitoneal administration of a combined CDDP, 5-FU and MMC therapy for pseudomyxoma peritonei]. [Article in Japanese]. Gan To Kagaku Ryoho. 1999 Oct;26(12):1828-31.

12. Sasaki A, Terashima M, Okamoto K, Ikeda K, Takagane A, Takiyama I, Kuboi M, Sasaki N, Saito K. [Two cases of pseudomyxoma peritonei that responded to intraperitoneal administrations of a combined CDDP, 5-FU and MMC therapy] [Article in Japanese]. Gan To Kagaku Ryoho. 1996 Sep;23(11):1571-4. 